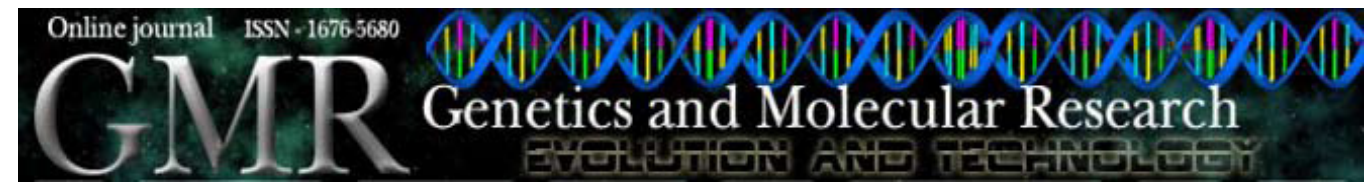

\title{
Glutathione S-transferase M1 and T1 polymorphism in men with idiopathic infertility
}

\author{
A.C.F. Finotti ${ }^{1,2}$, R.C.P. Costa e Silva ${ }^{1,2}$, B.M. Bordin ${ }^{1,2}$, C.T.X. Silva ${ }^{2}$ and \\ K.K.V.O. Moura ${ }^{1,2}$ \\ 'Programa de Pós-Graduação Stricto Sensu, Mestrado em Genética, Goiânia, \\ GO, Brasil \\ ${ }^{2}$ Departamento de Biologia, Núcleo de Pesquisas Replicon, Universidade \\ Católica de Goiás, Goiânia, GO, Brasil \\ Corresponding author: K.K.V.O. Moura \\ E-mail: kkverolli@ucg.br/katiakarinav@yahoo.com.br
}

Genet. Mol. Res. 8 (3): 1093-1098 (2009)

Received June 5, 2009

Accepted June 24, 2009

Published September 8, 2009

\begin{abstract}
Male infertility is a heterogeneous disorder, with various genetic and environmental factors that contribute to the impairment of spermatogenesis. Genetic causes are responsible for $60 \%$ of cases of idiopathic infertility. Polymorphisms of genes that encode detoxifying enzymes of phase II drug metabolism can modify their expression or function, affecting the biotransformation of toxic compounds to which the male reproductive system is exposed. GSTM1 and GSTT1 encode enzymes that are essential in the process of detoxification of endogenous and exogenous xenobiotics, facilitating their excretion. We examined GSTM1 and GSTT1 polymorphism in 233 men with idiopathic infertility seen at the Human Reproduction Service of the Federal University of Goiás from 2004-2006. Genotype GSTM1/T1 (null) was found in 30/105 normal individuals and in 64/128 abnormal individuals, indicating a significant association with idiopathic male infertility. The sperm alteration associated with greater GSTM1/T1 (null) frequency was decreased sperm count (oligozoospermy), which was more frequent in patients with GSTM1 $(78.9 \%)$ as well as in those who had GSTT1 (73.7\%), although the difference was not significant. Individuals polymorphic for genes GSTM1 and GSTT1 are susceptible to reduction in sperm quality and infertility, possibly because oligozoo-
\end{abstract}


spermic individuals have been affected by GST polymorphism.

Key words: GSTM1; GSTT1; Male infertility; Xenobiotics

\section{INTRODUCTION}

Infertility is a significant problem, affecting up to $15 \%$ of couples of reproductive age (Pasqualotto et al., 2004). Approximately $30 \%$ of men seeking treatment for their infertility present no demonstrable etiology and their condition remains idiopathic (Pasqualotto and Pasqualotto, 2006). It is a heterogeneous disorder, with several genetic and environmental factors contributing to impaired spermatogenesis (Aydemir et al., 2007). Studies revealed a significant decline in human semen quality and quantity over the past decades, and the increasing occurrence of disorders of the male reproductive tract is yet unknown. Environmental factors have been suggested to be implicated in the conditions of poor sperm function and infertility (Nakata et al., 2004; Rubes et al., 2007).

Xenobiotics constitute the majority of the environmental factors. They include naturally occurring compounds, drugs, environmental agents, and carcinogens. Adverse effects of xenobiotics are exerted via covalent interactions between intermediate metabolites and genetic components or proteins and their related metabolites (Nakata et al., 2004; Rubes et al., 2007).

Glutathione S-transferases (GSTs) constitute a superfamily of ubiquitous, multifunctional enzymes, which play a key role in phase II cellular detoxification and bioactivation reactions and are generally considered to be "antioxidant" enzymes (Schuppe et al., 2000; Hemachand and Shaha, 2003; Huber et al., 2008). These are widely expressed in mammalian tissues and have broad substrate specificity. GSTs are expressed in all living mammals and constitute more than $4 \%$ of the total soluble protein (Hatagima, 2002). Based on similarity of the amino acid sequence, GSTs have been grouped into at least seven classes known as alpha $(\alpha)$, mi $(\mu)$, pi $(\pi)$, theta $(\theta)$, zeta $(\zeta)$, sigma $(\sigma)$, and more recently discovered omega $(\Omega)$ (Coughlin and Hall, 2002).

Numerous genetic polymorphisms have been reported for GST enzymes (Hatagima, 2002; Joseph et al., 2006) resulting in a lack of functional protein or causing either increased or decreased metabolic activity (Rubes et al., 2007; Taspinar et al., 2008). Genes encoding the GST Mu-1 and Theta-1 (GSTM1 and GSTT1, respectively) isoforms are polymorphic (Chen et al., 2002; Nakata et al., 2004). Homozygotes for the mutated inactive alleles of each gene are devoid of any specific enzymatic activity of GSTM1 or T1 (null genotypes) (Schuppe et al., 2000).

Several epidemiological studies have reported that the GSTM1 and GSTT1 null genotypes are correlated with an increased susceptibility to diseases associated with oxidative stress and have proposed that GSTM1 and GSTT1 could be a critical isozyme in the detoxification of oxidative stress (Alvarez and Moraes, 2006; Aydemir et al., 2007). Although it has been shown that sperm surface GSTs are enzymatically active, the precise mechanism by which they protect spermatozoa is not clear (Chen et al., 2002; Aydemir et al., 2007).

The enzymatic deficiency of isoforms of GSTs has been correlated with increased risk to develop certain diseases associated with oxidative damage, such that an association between the genotypes GSTM1 and GSTT1 and the idiopathic infertility is possible. Because of the role of GSTs in inactivating endogenous end products formed as secondary metabolites during oxidative stress, the aim of this study was to examine the distribution of GSTM1 and GSTT1 polymorphisms in patients with idiopathic infertility. 


\section{MATERIAL AND METHODS}

\section{Study population}

Semen samples were collected from 233 patients (aged 15-69 years) at the Laboratory of Human Reproduction (HC-UFG) in 2004, 2005, and 2006. The patients were classified according to alterations detected with three consecutive spermograms, based on the WHO technique (1999), in two groups: normozoospermics and those showing alterations (abnormal spermogram). All subjects had a history of infertility with no indication of hormonal, infective, or physical causes for their infertility. The project was approved by the Ethics in Research Committee of the Catholic University of Goiás (\#150/2004), and a written informed consent form was signed by all participants.

\section{Molecular analysis}

Genomic DNA was extracted from semen with the Illustra ${ }^{\mathrm{TM}}$ Blood Genomic DNA kit (GE HealthCare, USA), in accordance with the recommended protocol optimized. The multiplex polymerase chain reaction (PCR) was used for amplification with the specific primer pairs for the genes GSTM1 (215 bp), GSTT1 (480 bp) and RH92600 (135 bp) (Chen et al., 2002). The PCR products were loaded onto $2 \%$ agarose gels for electrophoresis. After electrophoresis, the gels were stained with ethidium bromide and photographed under UV light (Image Master VDS ${ }^{\circledR}$ Amersham Pharmacia Biotech, USA) (Figure 1). Two bands of $480 \mathrm{bp}$ for GSTT1 and $215 \mathrm{bp}$ for GSTM1 were obtained for the M1/T1 genotype. The M0/T1 genotype showed a band of 480 $\mathrm{bp}$, and the M1/T0 genotype showed a band of $215 \mathrm{bp}$. For the M0/T0 genotype (homozygous absence or deletion genotype is designated as the null genotype), no bands were obtained and thus the use of an internal positive control (RH92600, $135 \mathrm{bp}$ ) was necessary to distinguish the null genotype from aborted PCR.

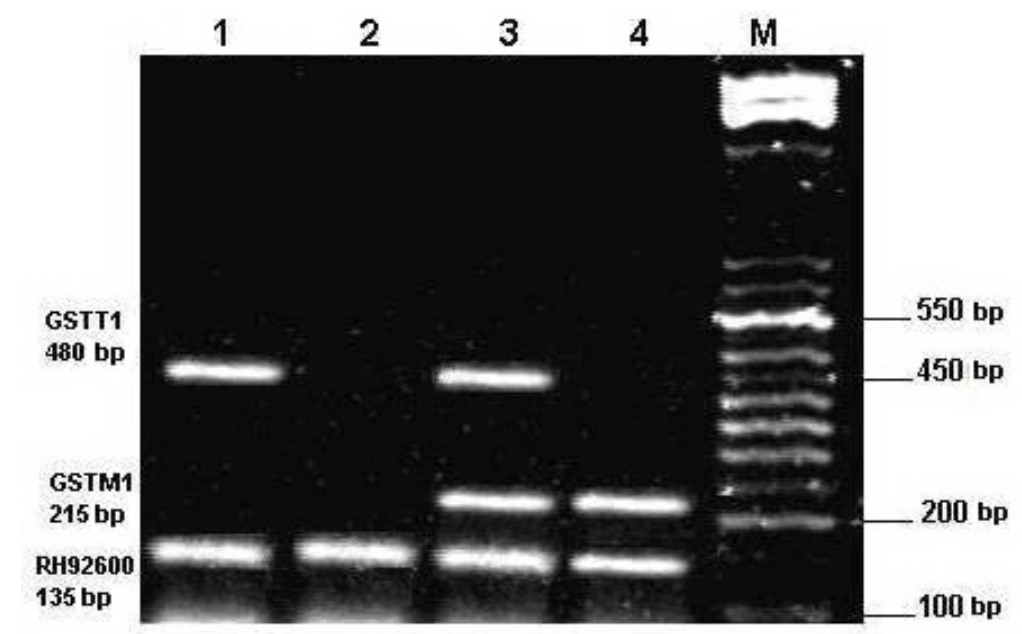

Figure 1. A $2 \%$ agarose gel showing polymerase chain reaction products of GSTM1 and GSTT1 polymorphism. Lane 1 = GSTT1 positive; lane $2=$ GSTM1/GSTT1 (null); lane $3=$ GSTM1/GSTT1 positive; lane $4=\mathrm{GSTM} 1$ positive; RH92600 = internal positive control; $\mathrm{M}=$ molecular weight ladder (50 bp). 
Statistical analysis for the possible associations between molecular analysis and the male idiopathic infertility was compared using the $\chi^{2}$ test. Results were considered to be significant when the probability of findings occurring by chance was less than $5 \%(\mathrm{P}<0.05)$. The BioEstat ${ }^{\mathbb{B}}$ 5.0 program (Sociedade Civil Mamirauá/MCT-CNPq) was used to carry out the statistical tests.

\section{RESULTS}

Of the 233 samples analyzed, the normal group made up $45.1 \%(105 / 233)$ and the group that showed alterations (abnormal spermogram) 54.9\% (128/233), where $29.7 \%$ (38) of the latter patients were oligozoospermics, $25 \%$ (32) asthenozoospermics, $32.1 \%$ (41) teratozoospermics, and $13.2 \%$ (17) azoospermics. The mean age of the patients with alterations was $34.66 \pm 7.46$ (mean $\pm \mathrm{SD}$ ), and for normal individuals this was $33.38 \pm 10.01$. Homogeneity between the two groups evaluated was established as $\mathrm{P}=0.096$.

Among the DNA samples analyzed, it was shown that the null genotypic frequency for the two genes GSTM1 and GSTT1 was $28.6 \%$ for the normal patients and $50 \%$ for the group that showed alterations. Positive genotype for both genes GSTM1 and GSTT1 was $29.5 \%$ for the normal patients and $23.4 \%$ for the abnormal group, which was found to be significantly different $(\mathrm{P}=0.008)$ (Table 1$)$.

$\begin{aligned} & \text { Table 1. Distribution of genotypic frequency in the semen samples of the patients from the normal and } \\
& \text { abnormal groups. }\end{aligned}$
\begin{tabular}{lccc}
\hline GSTM1/GSTT1 & Normal & Abnormal & P \\
\hline $0-0$ & $30(28.6 \%)$ & $64(50.0 \%)$ & 0.008 \\
$1-0$ & $10(9.5 \%)$ & $10(7.8 \%)$ & \\
$0-1$ & $34(32.4 \%)$ & $24(18.8 \%)$ & $30(23.4 \%)$ \\
$1-1$ & $31(29.5 \%)$ & $128(100 \%)$ & \\
Total & $105(100 \%)$ &
\end{tabular}

Data are reported as number with percent in parentheses. $0=$ null genotype; $1=$ positive genotype.

Based on the alterations of the spermogram, the null genotype for both genes GSTM1/ T1 was most frequent in oligozoospermics (39.1\%), and the positive genotype for both genes was more frequent in asthenozoospermics (36.7\%). Isolating the GSTM1 and GSTT1 genes, the null genotype appeared more frequently in oligozoospermic individuals for M1 (78.9\%) as well as for T1 $(73.7 \%)$, while the positive genotype was less frequent in azoospermics for M1 $(35.3 \%)$ as well as for $\mathrm{T} 1(47.1 \%)$; there was no statistically significant difference $(\mathrm{P}=0.350$ and $\mathrm{P}=0.132$, respectively) (Table 2 ).

\begin{tabular}{|c|c|c|c|c|c|c|c|c|c|}
\hline \multirow[t]{2}{*}{ Abnormal group } & \multicolumn{2}{|c|}{$\mathrm{M} 1 / \mathrm{T} 1$} & \multicolumn{2}{|c|}{$\mathrm{M} 1 / \mathrm{T} 1$} & \multicolumn{2}{|c|}{$\mathrm{M} 1 / \mathrm{T} 1$} & \multicolumn{2}{|c|}{$\mathrm{M} 1 / \mathrm{T} 1$} & \multirow[t]{2}{*}{$\mathrm{P}$} \\
\hline & $0-0$ & $\%$ & $1-0$ & $\%$ & $0-1$ & $\%$ & $1-1$ & $\%$ & \\
\hline Oligozoospermia & 25 & 39.1 & 3 & 30.0 & 5 & 20.8 & 5 & 16.7 & \\
\hline Asthenozoospermia & 14 & 21.9 & 2 & 20.0 & 5 & 20.8 & 11 & 36.7 & \\
\hline Teratozoospermia & 17 & 26.6 & 4 & 40.0 & 11 & 45.8 & 9 & 30.0 & 0.434 \\
\hline Azoospermia & 8 & 12.5 & 1 & 10.0 & 3 & 12.5 & 5 & 16.7 & \\
\hline Total & 64 & 100 & 10 & 100 & 24 & 100 & 30 & 100 & \\
\hline
\end{tabular}

$0=$ null genotype; 1 = positive genotype. 


\section{DISCUSSION}

In homozygosis, individuals with the null allele of the GSTM1 and GSTT1 genes are considered to be a risk group for various diseases, mainly if displaying elevated levels of carcinogens and noxious chemical compounds, because of the enzymatic defect in the detoxification system (Nakata et al., 2004).

Male infertility has been related to multiple irregularities including sperm concentration, motility and morphology (Huynh et al., 2002). Recent studies reported a significant decline in human semen quality or deterioration of spermatogenesis over the past decades (Fritsche et al., 1998; Alvarez and Moraes, 2006; Aydemir et al., 2007). Possible underlying reasons are yet unknown; however, xenobiotics have been suggested to exert adverse effects on the male reproductive system (Pasqualotto et al., 2008).

Although the influence of age on seminal quality has not yet been well defined, Pasqualotto et al. (2004) reported a decrease in sperm concentration after 45 years of age. Moskovtsev et al. (2006) in a study of 1125 infertile men demonstrated that damage to sperm DNA is significantly greater in men older than 40 years. The mean of age of the patients in our study did not show a significant difference compared to that of controls.

The polymorphism of GSTM1 and GSTT1 genes has been associated with male infertility (Schuppe et al., 2000) and also female infertility (Hadfield et al., 2001; Sata et al., 2003). When analyzing the genotype frequency within each group of normal and abnormal individuals from the semen sample, a statistically significant association was found between GST polymorphism and idiopathic male infertility. The most frequent genotype was GSTM1/T1 (null) in individuals with an abnormal spermogram, and oligozoospermia was more associated with the null polymorphism of the GSTM1 and GSTT1.

Chen et al. (2002) showed a correlation for elevated frequency of GSTM1 (null) and varicocele and subclinical varicocele patients when compared with the fertile group. Similarly, Alkan et al. (1997) indicated a greater predominance of GSTM1 (null) men and infertility; their spermograms pointed to oligozoospermia alone or in combination with asthenozoospermia or teratozoospermia. A possible explanation for sperm reduction in individuals with GSTM1/T1 (null) would be decreased capacity of seminiferous tubules and the increase in fibrosis of the testicular tissue, since the accumulation of xenobiotics not detoxified in the cellular matrix of the testis would impair spermatogenesis (Pajarinen et al., 1996).

A study in Turkey on infertile individuals according to criteria of the WHO indicated that there was no significant difference in genotypic frequency of GSTM1 (null) between patients of an infertile group (51.9\%) and a control group (46.7\%) (Aydemir et al., 2007). However, these authors verified the association between the polymorphism of gene GSTM1 and markers of oxidative stress in patients with idiopathic infertility.

In conclusion, the results of the present study suggest that the decline in the semen quality of individuals with idiopathic infertility is associated with the null genotype of glutathione S-transferase enzymes. Therefore, polymorphism of GSTM1/T1 hampers the important function of these enzymes in the biotransformation of xenobiotics, allowing them to affect the male reproductive system.

\section{ACKNOWLEDGMENTS}

Research supported by Universidade Católica de Goiás, Goiânia, Brazil (UCG/ PROPE/MGene). 


\section{REFERENCES}

Alkan I, Simsek F, Haklar G, Kervancioglu E, et al. (1997). Reactive oxygen species production by the spermatozoa of patients with idiopathic infertility: relationship to seminal plasma antioxidants. J. Urol. 157: 140-143.

Alvarez CA and Moraes GV (2006). Effect of the selenomethionine and vitamin C on the semen. SaBios: Rev. Saúde Biol. 1: $42-51$.

Aydemir B, Onaran I, Kiziler AR, Alici B, et al. (2007). Increased oxidative damage of sperm and seminal plasma in men with idiopathic infertility is higher in patients with glutathione S-transferase Mu-1 null genotype. Asian J. Androl. 9: $108-115$.

Chen SS, Chang LS, Chen HW and Wei YH (2002). Polymorphisms of glutathione S-transferase M1 and male infertility in Taiwanese patients with varicocele. Hum. Reprod. 17: 718-725.

Coughlin SS and Hall IJ (2002). Glutathione S-transferase polymorphisms and risk of ovarian cancer: a HuGE review. Genet. Med. 4: 250-257.

Fritsche E, Schuppe HC, Dohr O, Ruzicka T, et al. (1998). Increased frequencies of cytochrome P4501A1 polymorphisms in infertile men. Andrologia 30: 125-128.

Hadfield RM, Manek S, Weeks DE, Mardon HJ, et al. (2001). Linkage and association studies of the relationship between endometriosis and genes encoding the detoxification enzymes GSTM1, GSTT1 and CYP1A1. Mol. Hum. Reprod. 7: $1073-1078$.

Hatagima A (2002). Genetic polymorphisms and metabolism of endocrine disruptors in cancer susceptibility. Cad. Saúde Pública 18: 357-377.

Hemachand T and Shaha C (2003). Functional role of sperm surface glutathione S-transferases and extracellular glutathione in the haploid spermatozoa under oxidative stress. FEBS Lett. 538: 14-18.

Huber PC, Almeida WP and Fatima A (2008). Glutathione and related enzymes: biological roles and importance in pathological processes. Quim. Nova 31: 1170-1179.

Huynh T, Mollard R and Trounson A (2002). Selected genetic factors associated with male infertility. Hum. Reprod. Update. 8: 183-198.

Joseph T, Chacko P, Wesley R, Jayaprakash PG, et al. (2006). Germline genetic polymorphisms of CYP1A1, GSTM1, and GSTT1 genes in Indian cervical cancer: associations with tumor progression, age and human papillomavirus infection. Gynecol. Oncol. 101: 411-417.

Moskovtsev SI, Willis J and Mullen JB (2006). Age-related decline in sperm deoxyribonucleic acid integrity in patients evaluated for male infertility. Fertil. Steril. 85: 496-499.

Nakata LC, Goloni-Bertollo EM, Santos I, Oliani AH, et al. (2004). Biomarkers of susceptibility to endometriosis. Rev. Bras. Ginecol. Obstet. 26: 299-304.

Pajarinen J, Savolainen V, Perola M, Penttila A, et al. (1996). Glutathione S-transferase-M1 'null' genotype and alcoholinduced disorders of human spermatogenesis. Int. J. Androl. 19: 155-163.

Pasqualotto FF and Pasqualotto EB (2006). Treatment the infertile man with varicocele. J. Bras. Med. 90: 38-46.

Pasqualotto FF, Lucon AM, Sobreiro BP, Pasqualotto EB, et al. (2004). Effects of medical therapy, alcohol, smoking, and endocrine disruptors on male infertility. Rev. Hosp. Clin. Fac. Med. São Paulo 59: 375-382.

Pasqualotto FF, Umezu FM, Salvador M, Borges E Jr, et al. (2008). Effect of cigarette smoking on antioxidant levels and presence of leukocytospermia in infertile men: a prospective study. Fertil. Steril. 90: 278-283.

Rubes J, Selevan SG, Sram RJ, Evenson DP, et al. (2007). GSTM1 genotype influences the susceptibility of men to sperm DNA damage associated with exposure to air pollution. Mutat. Res. 625: 20-28.

Sata F, Yamada H, Kondo T, Gong Y, et al. (2003). Glutathione S-transferase M1 and T1 polymorphisms and the risk of recurrent pregnancy loss. Mol. Hum. Reprod. 9: 165-169.

Schuppe HC, Wieneke P, Donat S, Fritsche E, et al. (2000). Xenobiotic metabolism, genetic polymorphisms and male infertility. Andrologia 32: 255-262.

Taspinar M, Aydos SE, Comez O, Elhan AH, et al. (2008). CYP1A1, GST gene polymorphisms and risk of chronic myeloid leukemia. Swiss. Med. Wkly. 138: 12-17.

World Health Organization (WHO) (1999). Laboratory Manual for the Examination of Human Semen and Semen-Cervical Mucus Interaction. 3rd edn. Cambridge University Press, New York. 